\title{
Editorial
}

\section{Ion channels research in the post-genomic era}

\author{
Bernard ATTALI ${ }^{1, *}$, Zhao-bing GAO ${ }^{2, *}$ \\ ${ }^{1}$ Department of Physiology \& Pharmacology, Sackler Faculty of Medicine, Sagol School of Neuroscience, Tel Aviv University, Tel Aviv \\ 69978, Israel; ${ }^{2}$ CAS Key Laboratory of Receptor Research, Shanghai Institute of Materia Medica, Chinese Academy of Sciences, \\ Shanghai 201203, China
}

Acta Pharmacologica Sinica (2016) 37: 1-2; doi: 10.1038/aps.2015.144

Ion channels are integral membrane proteins that catalyze the flux of ions across cell membranes, and play fundamental roles in a wide variety of functions throughout the body ${ }^{[1]}$. Thus, in the nervous system, various ion channels concert in regulating neuronal excitability, shaping action potentials, tuning neuronal firing patterns, and controlling transmitter release or synaptic integration. In the cardiovascular system, they control electrical excitability, impulse conduction and muscle contraction. In the kidney they regulate fluid secretion and electrolyte balance; and in the immune system, they drive exocytosis, stimulate mitochondrial metabolism, activate gene expression, and promote the activation, growth and proliferation of T cells. Moreover, ion channels also act as regulators in developmental processes and tissue patterning, and disruption of their function affects morphogenesis in flies, fishes, frogs and mammals. On the other hand, ion channels play essential roles in the pathophysiology of various diseases, and it is therefore not surprising that an ever-growing number of diseases are found to be caused by dysfunction of ion channels. Genetic defects in ion channels lead to severe neurological, cardiac, muscular or metabolic disorders, which are called 'channelopathies' ${ }^{[1]}$. As such, ion channels are recognized as important drug targets. In fact, a number of small molecule modulators of ion channels have been used clinically as antiarrhythmic, anti-hypertensive, anti-epileptic or diuretic agents for decades.

In this post-genomic era, when the genes encoding most ion channels have been deciphered with their function remaining elusive yet, a full exploration of the channel proteins has become a new frontier of biological and biomedical research. Despite their well-known importance, the basic structural and physiological properties of ion channels are far from being

\footnotetext{
* To whom correspondence should be addressed.

E-mail battali@post.tau.ac.il (Bernard ATTALI); zbgao@simm.ac.cn (Zhao-bing GAO)
}

understood, but have recently become approachable due to the development of new technologies, thereby enabling their purification, modification, functional and structural analyses. It is now clear that ion channel proteins operate within macromolecular structures and signaling complexes whose study presents an exceptional challenge for scientists. Nowadays, ion channel research is undergoing exciting times full of crystal structure determinations, pharmacological targeting and spectroscopic studies of protein motions. Precisely at these times, there is an urgent need to integrate the interplay of structural, biophysical, pharmacological, and physiological studies. The articles in this special issue "Ion Channel Research in PostGenomic Era" reflect such an effort to integrate the different aspects of this experimental and conceptual challenge.

TRP channels represent a large protein family with more than 30 distinct subtypes, which are involved in various biological processes such as sensory transduction, calcium absorption, neuronal growth cone guidance, keratinocyte development, etc. TRP channel-associated channelopathies lead to a wide range of diseases like polycystic kidney disease, skeletal dysplasia, and familial episodic pain syndrome. Thus, understanding how TRP channels respond to physiological stimuli and drugs is of direct clinical and therapeutic relevance to these diseases. In this special APS issue, Zhan et $a l^{[2]}$ examine how TRPM2, acting as a reactive oxygen species sensor and $\mathrm{Ca}^{2+}$-permeable ion channel, is involved in ischemia/reperfusion injury. They discuss the recent progress in understanding the role of TRPM2-mediated ischemia/reperfusion injury in brain, heart and kidney, and the therapeutic potentials of targeting TRPM2 for drug development. Du et $a l^{[3]}$ provide an overview of TRPP2 channel protein and its possible role in cardiovascular function and diseases. TRPP2 channel (polycystin-2, or PKD2) is located in the membrane of endoplasmic reticulum, and mediates intracellular $\mathrm{Ca}^{2+}$ release associated with $\mathrm{IP}_{3}$ receptors and ryanodine receptors. The mutation of PKD2 gene is one major cause of autosomal dominant polycystic kidney disease (ADPKD), associated with abnormalities in the vasculature and cardiovascular complica- 
tions. He and $\mathrm{Ma}^{[4]}$ focus on TRPC5 channels and its role in cancer. Dysregulation of TRPC5 interrupts $\mathrm{Ca}^{2+}$ homeostasis in cancer cells, which activates the signaling pathways related with cancer progression, especially cancer chemoresistance. They also discuss the potential of TRPC5 as a target for therapeutic intervention. Tang et $a l^{[5]}$ examine how the regulatory protein Pirt regulates TRPM8 channels $\left(\mathrm{a} \mathrm{Ca}^{2+}\right.$-permeable nonselective cation channel activated by menthol and cold below $25^{\circ} \mathrm{C}$, and expressed in dorsal roots and trigeminal ganglia). They demonstrate that Pirt enhances TRPM8 channel activity by improving the channel conductance, and Pirt combined with PIP2 exhibits a synergistic regulatory effect on TRPM $8^{[5]}$.

KCNQ channels (Kv7.1-5 or KCNQ1-5) comprise a family of voltage-gated $\mathrm{K}^{+}$channels located in brain, heart, kidney, stomach, pancreas and inner ear. Kv7.1 channels, in conjunction with the auxiliary $\beta$ subunit KCNE1, generate the $I_{\mathrm{KS}}$ current crucial for the repolarization of cardiac action potentials. Heteromultimeric Kv7.2/7.3 channels produce the characteristically slowly-activating, non-inactivating neuronal $I_{\mathrm{M}}$ current that operates in the subthreshold range of the action potential, thereby modulating neuronal excitability. Mutations in human Kv7.1-7.4 genes are associated with major cardiovascular and neurological disorders such as cardiac arrhythmias (the long QT syndrome), and neonatal epilepsy or deafness. In this special APS issue, Wang and $\mathrm{Li}^{[6]}$ discuss the roles of KCNQ channels in sensory system and neural circuits, and their regulatory mechanisms and implications for physiology and medicine. They also present future perspectives to the development of novel drugs targeting KCNQ channels for the treatment of neurological diseases, such as seizure, stroke, pain and mental illness. Yue et $a l^{[7]}$ show that thallium flux assay combined with the IonWorks Barracuda system is an efficient high-throughput screening (HTS) route for identifying KCNQ2 openers. They discovered $38 \mathrm{KCNQ} 2$ activators from a collection of 80000 compounds, and 2 of them with $\mathrm{EC}_{50}$ values at micromolar level ${ }^{[7]}$. Along this approach, $\mathrm{Yu}$ et al summarize the HTS technology used in pharmaceutical industry for diverse ion channel classes and cell types ${ }^{[8]}$. More efforts are being made to improve the capability of automated electrophysiological instruments for hERG-related drug-safety screening ${ }^{[9]}$. Xie et al ${ }^{[10]}$ investigated the effects of 1-alkanols on cardiac KCNQ1 channels. They show that ethanol, 1-butanol and 1-hexanol all inhibit $I_{\mathrm{KS}}$ (KCNQ1/KCNE1) currents with longer chain 1-alkanols causing more inhibition, and the residue (I257) in the intracellular loop between S4 and S5 in KCNQ1 is crucial for the sensitivity to 1-alkanols in a closedstate channel block ${ }^{[10]}$.

In addition to TRP and KCNQ channels, recent progresses and future directions of multiple ion channels are also described in this special APS issue. In order to understand the basis of the pharmacology of $\mathrm{K}_{\mathrm{ATP}}$ channel openers against metabolic disorders, Li et al investigated the responses of $\mathrm{K}_{\text {ATP }}$ channels to metabolic inhibitor Na-azide ${ }^{[11]}$. Wang and $\mathrm{Yu}^{[12]}$ review the general knowledge on the structure-function relationship of P2X receptors. Yu et al ${ }^{[13]}$ provide an overview of the structural and pharmacological properties of BK channel blockers. Ge and $\mathrm{Liu}^{[14]}$ discuss the prevailing hypotheses attributing the electrical-resonance to voltage-gated ion channels on neuronal membrane. Stoler and Fleidervish ${ }^{[15]}$ present a brief overview of the structure and function of axon initial segment (AIS) and discuss the roles of calpains and possible sources of $\mathrm{Ca}^{2+}$ ions in the stroke-induced AIS damage. Finally, Weisbrod et al ${ }^{[16]}$ discuss the various ionic mechanisms underlying the pacemaker activity. They also describe the latest characterization of the previously unrecognized role of the SK4 $\mathrm{Ca}^{2+}$-activated $\mathrm{K}^{+}$channel conductance in pacemaker cells. By opposing to inward currents during the diastolic depolarization, the SK4 channels appear to play a crucial role in human cardiac automaticity.

In conclusion, ion channel study is a multidisciplinary research embracing various fields of science, including structural and cell biology, pharmacology, biophysical and physiological approaches, as well as chemical and computational design strategies, which is providing a better understanding of the pathophysiology of ion channel-related diseases, and clues to treat them. The future of this field is very promising, and we wish that the gathering of these articles would provide new insights into ion channel research and drug discovery.

\section{References}

1 Ashcroft FM. Ion channels and diseases. Academic Press, 2000.

2 Zhan KY, Yu PL, Liu CH, Luo JH, Yanf W. Detrimental or beneficial: the role of TRPM2 in ischemia/reperfusion injury. Acta Pharmacol Sin 2016; 37: 1-2.

3 Du J, Fu J, Xia XM, Shen B. The function of TRPP2 in the vascular system. Acta Pharmacol Sin 2016; 37: 1-2.

4 He DX, Ma X. Transient receptor potential channel C5 in chemoresistance. Acta Pharmacol Sin 2016; 37: 1-2.

5 Tang M, Wu GY, Dong XZ, Tang ZX. The regulatory mechanism of Pirt enhancing TRPM8 channel excitability. Acta Pharmacol Sin 2016; 37: $1-2$.

6 Wang JJ, Li Y. KCNQ potassium channels in neuronal circuits. Acta Pharmacol Sin 2016; 37: 1-2.

7 Yue JF, Qiao GH, Liu N, Chen XQ, Nan FJ, Gao ZB. Identifying novel KCNQ2 activators using fluorescence-based and automated patchclamp-based high-throughput assays. Acta Pharmacol Sin 2016; 37: $1-2$.

$8 \mathrm{Yu}$ HB, Li M, Wang WP, Wang XL. High throughput screening technologies for ion channels. Acta Pharmacol Sin 2016; 37: 1-2.

9 Yu HB, Zou BY, Wang XL, Li M. Investigation of promiscuous hERG inhibition by a large diverse compound collection using automated electrophysiology. Acta Pharmacol Sin 2016; 37: 1-2.

10 Xie C, Liu HW, Pan N, Ding JP, Yao J. Identification of 1257 in the S4S5 linker responsible for the closed-state block of KCNQ1 channels by 1-alkanols. Acta Pharmacol Sin 2016; 37: 1-2.

11 Li CG, Cui WY, Wang H. Sensitivity of SUR/Kir6.x channels to cellular metabolic inhibition and the underlying structural basis. Acta Pharmacol Sin 2016; 37: 1-2.

12 Wang J, Yu Y. Insights into channel gating of P2X receptors from structures, dynamics and small molecules. Acta Pharmacol Sin 2016; 37: 1-2.

13 Yu M, Liu SL, Sun PB, Pan H, Tian CL, Zhang LH. Peptide Toxins and Small Molecule Blockers for the BK Channel. Acta Pharmacol Sin 
2016; 37: 1-2.

14 Liu XD, Ge L. Electrical resonance with voltage-gated ion Channels: perspectives from biophysical mechanisms and neural electrophysiology. Acta Pharmacol Sin 2016; 37: 1-2.

15 Stoler O, Fleidervish IA. Functional Implications of Axon Initial
Segment Cytoskeletal Disruption in Stroke. Acta Pharmacol Sin 2016; 37: 1-2.

16 Weisbrod D, Khun SH, Bueno H, Peretz A, Attali B. Mechanisms underlying the cardiac pacemaker: role of the SK4 calcium-activated potassium channel. Acta Pharmacol Sin 2016; 37: 1-2. 